\title{
Exposure-response relationship of ramucirumab in patients with advanced second-line colorectal cancer: exploratory analysis of the RAISE trial
}

\author{
Allen Lee Cohn ${ }^{1} \cdot$ Takayuki Yoshino $^{2} \cdot$ Volker Heinemann $^{3} \cdot$ Radka Obermannova $^{4} \cdot$ György Bodoky $^{5}$. \\ Jana Prausová $^{6} \cdot$ Rocio Garcia-Carbonero $^{7} \cdot$ Tudor Ciuleanu $^{8} \cdot$ Pilar Garcia-Alfonso $^{9} \cdot$ David C. Portnoy $^{10}$. \\ Eric Van Cutsem ${ }^{11}$ - Kentaro Yamazaki ${ }^{12}$ Philip R. Clingan ${ }^{13}$ • Jonathon Polikoff ${ }^{14}$ • Sara Lonardi ${ }^{15}$. \\ Lisa M. O'Brien ${ }^{16} \cdot$ Ling Gao $^{17} \cdot$ Ling Yang $^{17} \cdot$ David Ferry $^{17} \cdot$ Federico Nasroulah $^{18} \cdot$ Josep Tabernero $^{19}$
}

Received: 5 April 2017 / Accepted: 27 June 2017 / Published online: 25 July 2017

(c) The Author(s) 2017. This article is an open access publication

\begin{abstract}
Purpose To characterize ramucirumab exposure-response relationships for efficacy and safety in patients with metastatic colorectal cancer (mCRC) using data from the RAISE study.

Methods Sparse pharmacokinetic samples were collected; a population pharmacokinetic analysis was conducted. Univariate and multivariate Cox proportional hazards models analyzed the relationship between predicted ramucirumab minimum trough concentration at steady state $\left(C_{\text {min,ss }}\right)$ and survival. Kaplan-Meier analysis was used to evaluate survival from patients in the ramucirumab plus folinic acid, 5-fluorouracil, and irinotecan (FOLFIRI) treatment arm stratified by $C_{\text {min,ss }}$ quartiles (Q). An ordered categorical
\end{abstract}

Electronic supplementary material The online version of this article (doi:10.1007/s00280-017-3380-z) contains supplementary material, which is available to authorized users.

Allen Lee Cohn

allen.cohn@usoncology.com

Rocky Mountain Cancer Center, 1800 Williams Street, Denver, CO 80218, USA

2 National Cancer Center Hospital East, Chiba, Japan

3 Ludwig-Maximilians-University Hospital Munich, Munich, Germany

4 Masaryk Memorial Cancer Institute, Brno, Czech Republic

5 Szent László Hospital, Budapest, Hungary

6 University Hospital Motol, Prague, Czech Republic

7 Hospital Virgen del Rocio, Seville, Spain

8 Institutul Oncologic Cluj-Napoca, Cluj-Napoca, Romania

9 Hospital General Universitario Gregorio Marañón, Madrid, Spain model analyzed the relationship between $C_{\text {min,ss }}$ and safety outcomes.

Results Pharmacokinetic samples from 906 patients were included in exposure-efficacy analyses; samples from 905 patients were included in exposure-safety analyses. A significant association was identified between $C_{\mathrm{min}, \mathrm{ss}}$ and overall survival (OS) and progression-free survival (PFS) $(p<0.0001$ for both). This association remained significant after adjusting for baseline factors associated with OS or PFS ( $p<0.0001$ for both). Median OS was 11.5, 12.9, 16.4, and 16.7, and 12.4 months for ramucirumab $C_{\text {min,ss }}$ Q1, Q2, Q3, Q4, and placebo group, respectively. Median PFS was 5.4, 4.6, 6.8, 8.5, and 5.2 months for ramucirumab $C_{\text {min,ss }}$ Q1, Q2, Q3, Q4, and placebo group, respectively. The risk of Grade $\geq 3$ neutropenia was associated with an increase in ramucirumab exposure.

Conclusions Exploratory exposure-response analyses suggested a positive relationship between efficacy

10 West Clinic, Memphis, TN, USA

11 University Hospitals Gasthuisberg, Louvain, Belgium

12 Shizuoka Cancer Center, Shizuoka, Japan

13 Southern Medical Day Care Centre, Wollongong, Australia

14 Kaiser Permanente San Diego, San Diego, CA, USA

15 Istituto Oncologico Veneto IOV - IRCCS, Padua, Italy

16 Eli Lilly and Company, Indianapolis, IN, USA

17 Eli Lilly and Company, Bridgewater, NJ, USA

18 Eli Lilly and Company, Buenos Aires, Argentina

19 Vall d'Hebron University Hospital and Institute of Oncology (VHIO), Barcelona, Spain 
and ramucirumab exposure with manageable toxicities in patients from the RAISE study with mCRC over the ranges of exposures achieved by a dose of $8 \mathrm{mg} / \mathrm{kg}$ every 2 weeks in combination with FOLFIRI.

Keywords Ramucirumab - Exposure-response · Colorectal cancer $\cdot$ Second line $\cdot$ FOLFIRI

\section{Introduction}

Colorectal carcinoma (CRC) is the third leading cause of cancer worldwide [1] and ranks fourth among leading causes of cancer deaths worldwide [2]. Conventional systemic therapy for CRC includes fluoropyrimidine-based regimens alone or in combination with irinotecan or oxaliplatin [3-7]. The development of agents targeting the epidermal growth factor receptor (EGFR) and angiogenic pathways has provided additional treatment options. Vascular endothelial growth factor (VEGF) and VEGF receptor-2 (VEGFR-2)-mediated signaling and angiogenesis are important in CRC tumor growth and are established therapeutic targets. Ramucirumab is a human IgG1 monoclonal antibody that specifically binds to the extracellular domain of VEGFR-2 with high affinity, preventing binding of VEGF$\mathrm{A}, \mathrm{C}$, and D ligands and receptor activation [8]. The safety and efficacy of ramucirumab in combination with secondline folinic acid, 5 fluorouracil, and irinotecan (FOLFIRI) in patients with metastatic CRC that progressed during or after first-line therapy with bevacizumab, oxaliplatin, and a fluoropyrimidine were evaluated in a randomized, doubleblind, placebo-controlled phase III trial (RAISE) [9]. On Day 1 of each 2-week cycle, patients received either $8 \mathrm{mg}$ / $\mathrm{kg}$ ramucirumab or placebo as a $60 \mathrm{~min}$ intravenous infusion, followed by the FOLFIRI regimen. The RAISE trial demonstrated a statistically significant survival benefit for patients treated with ramucirumab plus FOLFIRI versus placebo plus FOLFIRI with a median overall survival (OS) of 13.3 months (95\% confidence interval [CI] 12.4-14.5) for patients in the ramucirumab group versus 11.7 months (95\% CI 10.8-12.7) for the placebo group (hazard ratio [HR] $0.844,95 \%$ CI $0.730-0.976$; log-rank $p=0.0219$ ). Ramucirumab plus FOLFIRI was well tolerated and the adverse events were considered manageable [9].

All drugs have dose effect curves, a threshold concentration below which they are ineffective, a concentration where effect has reached a maximum plateau and between these extremes a range where increasing exposure increases effectiveness. The 'exposure-response' phenomenon occurs in the range of concentrations (exposure) where increasing exposure correlates with increasing effect. The phenomenon of exposure-response is seen with many antibodies in the treatment of cancer, including ipilimumab in melanoma
[10], trastuzumab emtansine in breast cancer [11], rituximab in the treatment of low-grade B cell malignancies, and rilotumumab in gastric cancer [12]. Analyses of the exposure-response relationship of ramucirumab in patients with gastric cancer (REGARD and RAINBOW trials) and non-small cell lung cancer (REVEL trial) have previously been reported [13, 14]. In both RAINBOW and REGARD, higher exposure to ramucirumab was associated with longer OS and progression-free survival (PFS) for gastric cancer patients. In REVEL, higher exposure to ramucirumab was associated with longer OS and PFS for non-small cell lung cancer patients.

The objective of this exploratory analysis was to determine whether there is an exposure-response relationship for ramucirumab in patients with advanced CRC enrolled in the RAISE trial.

\section{Materials and methods}

The details of the RAISE trial including patient eligibility, trial design, randomization, dose administration, clinical outcome definitions, and statistical analyses have been previously published [9]. Each center's institutional review board or independent ethics committee approved the study. The trial followed the principles of the Declaration of Helsinki and the Good Clinical Practice Guidelines of the International Conference on Harmonisation. All patients provided written informed consent. To summarize, eligible patients included those with pathologically confirmed metastatic CRC, known KRAS exon 2 mutation status (mutant or wild-type), and an Eastern Cooperative Oncology Group performance status (ECOG PS) score of 0 or 1 with disease progression during or within 6 months of the last dose of first-line combination therapy with bevacizumab, oxaliplatin, and a fluoropyrimidine for metastatic disease. Patients were randomized (1:1) to receive on Day 1 of each 2 -week cycle either $8 \mathrm{mg} / \mathrm{kg}$ ramucirumab or placebo as a $60 \mathrm{~min}$ intravenous infusion, followed by the FOLFIRI regimen $\left(180 \mathrm{mg} / \mathrm{m}^{2}\right.$ intravenous irinotecan given over $90 \mathrm{~min}$ followed by or concurrent with $400 \mathrm{mg} /$ $\mathrm{m}^{2}$ intravenous leucovorin given over $120 \mathrm{~min}$, followed by $400 \mathrm{mg} / \mathrm{m}^{2}$ fluorouracil given as an intravenous bolus over $2-4 \mathrm{~min}$ then $2400 \mathrm{mg} / \mathrm{m}^{2}$ given as a continuous infusion over 48 h; Fig. 1). Dose modifications were permitted for ramucirumab in the setting of non-life-threatening and reversible Grade 3 clinical adverse events (AEs; for example, fever) considered to be at least possibly related to investigational product and that resolved to Grade $\leq 1$ or pre-treatment baseline within 1 treatment cycle (approximately 2 weeks). FOLFIRI modifications were allowed for each component and were based on toxicities observed 
Metastatic colorectal carcinoma (mCRC) progression during or after bevacizumab, oxaliplatin, and a fluoropyrimidine

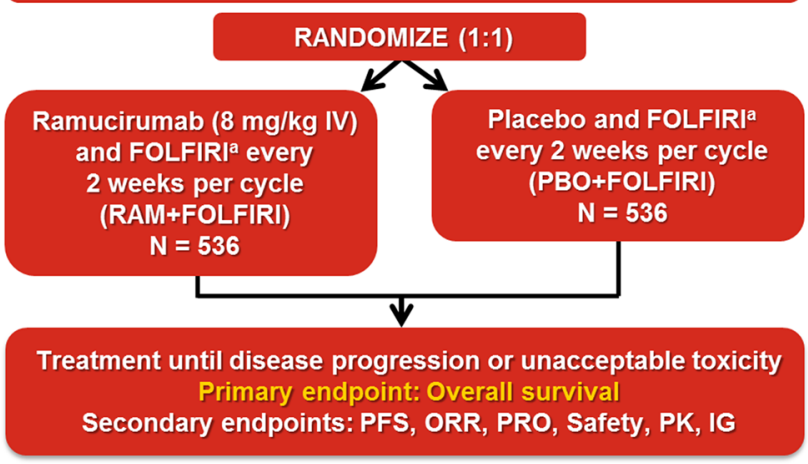

Patient enrollment: Dec.14, 2010 - Aug. 23, 2013

Fig. 1 RAISE study design ${ }^{a}$ Irinotecan: $180 \mathrm{mg} / \mathrm{m}^{2}$; Folinic acid: $400 \mathrm{mg} / \mathrm{m}^{2}$; 5-flurouracil: $400 \mathrm{mg} / \mathrm{m}^{2}$ bolus followed by $400 \mathrm{mg} /$ $\mathrm{m}^{2}$ given as a continuous infusion over 48 hours FOLFIRI, folinic acid, 5-fluorouracil, and irinotecan; $I G$ immunogenicity; $I V$ intravenous; $O R R$ objective response rate; $P F S$ progression-free survival; $P K$ pharmacokinetics; $P R O$ patient-reported outcomes; $R A M$ ramucirumab

and graded according to the National Cancer Institute Common Terminology Criteria for Adverse Events (NCICTCAE) v4.0.

The primary endpoint was OS, defined as the time from randomization to death from any cause. Key secondary endpoints included PFS (defined as time from randomization to progressive disease or death, whichever occurred first), the proportion of patients who achieved an objective response (defined as complete response or partial response), pharmacokinetic (PK) parameters of ramucirumab, and safety.

Pre-dose and 1-h post-infusion PK samples to determine ramucirumab serum concentration were collected at the following timepoints per study protocol: Day 1 of Cycles 3, 5, 9, 13, and 17. Due to the timing of PK sample collection, only patients from both treatment arms who had non-missing concentration data and who did not die or discontinue prior to Day 1 Cycle 3 were included in the exposure-response analysis. The patients included in the exposure-response analyses are a non-random subset of the intent-to-treat (ITT) population.

Serum ramucirumab concentration was determined using a validated enzyme-linked immunosorbent assay (ELISA) at Intertek Pharmaceutical Services (San Diego, CA, USA). A population $\mathrm{PK}$ model was developed using a nonlinear mixed-effect modeling approach (NONMEM 7.3 [Icon, Ellicott City, MD]) and in accordance with the U.S. Food and Drug Administration (FDA) Guidance for Industry on Population Pharmacokinetics [15, 16]. Population PK modelpredicted minimum concentration at steady state $\left(C_{\text {min,ss }}\right)$ was used to assess the exposure-response relationship.

\section{Exposure-efficacy analysis}

The exposure-efficacy analysis was conducted using SAS ${ }^{\circledR}$ version 9.1.2 or higher. Univariate and multivariate Cox proportional hazards models were used to evaluate the relationship between ramucirumab exposure and efficacy endpoints (OS and PFS). Data for the ramucirumab plus FOLFIRI treatment arm were separated into four quartiles $(\mathrm{Q})$ based on the exposure parameter of interest, $C_{\text {min,ss }}$. Kaplan-Meier analyses were performed for OS and PFS with data from patients in the ramucirumab plus FOLFIRI arm stratified according to $C_{\text {min,ss }}$ quartile; each quartile was compared with the data from patients in the placebo plus FOLFIRI (control) arm. A multivariate Cox model adjusted for baseline covariates was used to estimate the HR for each quartile versus the control arm. Stepwise Cox regression, with entry $p$ value $=0.05$ and exit $p$ value $=0.10$, selected the baseline factors prognostic for OS or PFS. These significant factors were used to adjust the final model for evaluating exposure-efficacy relationships. An additional matched case-control analysis for OS was explored to adjust for the potential imbalances in important prognostic factors between treatment arms within each exposure quartile group [17]. In this analysis, the case groups are the four exposure quartiles of $C_{\text {min,ss }}$ in the ramucirumab plus FOLFIRI arm. For every patient in each case group, a matched control patient was identified from all patients receiving placebo plus FOLFIRI, through a matching scheme based on the six significant potential prognostic factors identified in the stepwise Cox regression analysis and two additional covariates with the largest baseline imbalance in the subset of patients for this analysis (sex and prior bevacizumab use-composite subgroup). The two additional factors were selected based on a stepwise logistic regression of treatment arm assignment on the same pool of covariates in the stepwise Cox regression, and stepwise selection using entry and exit $p$ values of 0.2 (since randomization is supposed to make treatment assignment to be independent of baseline variables, a larger significance level was used to pick up any imbalanced factors due to chance). Missing values in any of the matching factors excluded the patients from the matched case-control study.

\section{Exposure-safety analysis}

Ordered categorical and logistic regression models were developed to explore the relationship between ramucirumab exposure $\left(C_{\mathrm{min}, \mathrm{ss}}\right)$ and the safety endpoints. Safety endpoints for exposure-safety analysis were the three most common Grade $\geq 3$ treatment-emergent adverse events (TEAEs) in the ITT population occurring in $\geq 5 \%$ of patients in the ramucirumab plus FOLFIRI arm, with a difference in incidence rate between the ramucirumab arm and the placebo arm of $\geq 2 \%$. Neutropenia, hypertension, and fatigue were the 
selected endpoints for exposure-safety analysis based on these criteria. For this analysis, neutropenia and fatigue are consolidated terms (composite terms consisting of multiple related preferred terms) based on Standardized MedDRA ${ }^{\circledR}$ Queries (SMQ) and medical review. Diarrhea was included as an adverse event of interest as it is one of the most frequent TEAE for the FOLFIRI regimen [18, 19]. Safety endpoints were graded per the NCI-CTCAE v4.0.

\section{Results}

Data from a total of 425 patients from the ramucirumab plus FOLFIRI arm and 481 patients from the placebo plus FOLFIRI arm were included in the exposure-efficacy analyses; data from a total of 425 patients from the ramucirumab plus FOLFIRI arm and 480 patients from the placebo plus FOLFIRI arm were included in the exposure-safety analyses. One patient randomized to the placebo group received ramucirumab as the first dose and was included in the placebo plus FOLFIRI arm for exposure-efficacy analysis and excluded from the placebo plus FOLFIRI arm for exposure-safety analysis.

An exploratory Cox regression analysis identified a statistically significant positive association between OS and $C_{\text {min,ss }}$ in a univariate analysis $(p<0.0001)$. A multivariate Cox regression analysis was used to adjust for the factors that were significantly associated with OS: time to progression after beginning first-line therapy, KRAS status, ECOG PS, number of metastatic sites, liver only metastasis, and carcinoembryonic antigen (CEA). After adjusting for these baseline factors, the association between OS and $C_{\text {min,ss }}$ remained statistically significant $(p<0.0001)$. Similar to OS, a statistically significant positive association was identified between PFS and $C_{\text {min,ss }}$ in a univariate Cox regression analysis $(p<0.0001)$. A multivariate Cox regression analysis was used to adjust for the factors that were significantly associated with PFS: ECOG PS, number of metastatic sites, liver only metastasis, CEA, and prior bevacizumab use (composite subgroup). A similar association between ramucirumab exposure and PFS was observed after adjusting for these significant baseline factors $(p<0.0001)$.

Ramucirumab concentration data from patients in the ramucirumab plus FOLFIRI arm were grouped into four $C_{\text {min,ss }}$ quartiles ( $<25 \%$ [Q1], $25 \%$ to $<50 \%$ [Q2], $50 \%$ to $<75 \%$ [Q3], and $\geq 75 \%$ [Q4]) based on the predicted $C_{\text {min,ss }}$ concentration. Demographic data and baseline disease characteristics by $C_{\text {min,ss }}$ quartile are presented in Table 1. Similarity in the data in Supplemental Table 2 between the overall study population (ITT population) [9] and the exposure-response population suggests that the analyzed subpopulation is reflective of the overall population enrolled in RAISE.
The Kaplan-Meier plots of OS and PFS by $C_{\text {min,ss }}$ quartiles are presented in Fig. 2a and b. Separation between the OS curves was observed among the four exposure groups, suggesting that higher exposure may be associated with longer OS time. Median OS was 11.5, 12.9, 16.4, and 16.7 months for the ramucirumab $C_{\text {min,ss }}$ Q1, Q2, Q3, and Q4 groups, respectively (Table 2). Median OS in the placebo plus FOLFIRI arm was 12.4 months (Table 2). Hazard ratios by quartile were adjusted for baseline factors. There was a trend of numerically decreasing hazard ratios as the predicted concentration of ramucirumab $C_{\text {min,ss }}$ increased. Quartile 1 and Q2 were not significantly different from the placebo group; however, Q3 and Q4 showed hazard ratios favoring the ramucirumab plus FOLFIRI treatment arm (Table 2).

The Kaplan-Meier plots of PFS curves were similar to those for OS (Fig. 2b). The higher exposure groups were associated with longer PFS. Median PFS was 5.4, 4.6, 6.8, and 8.5 months for the ramucirumab $C_{\text {min,ss }}$ Q1, Q2, Q3, and Q4 groups, respectively (Table 2). Median PFS in the placebo plus FOLFIRI arm was 5.2 months (Table 2). The PFS hazard ratios were adjusted for baseline factors and decreased as the concentration of predicted ramucirumab $C_{\text {min,ss }}$ increased by quartile. Similar to the results for OS, PFS for Q1 and Q2 were not significantly different from the placebo group, but Q3 and Q4 demonstrated progressively decreasing HRs that were significantly different from the placebo group.

A matched case-control analysis for OS was explored to evaluate the exposure-OS relationship and adjust for imbalances between the $C_{\text {min,ss }}$ quartiles. As previously described, there were eight matching factors for which outcomes were to be adjusted: time to progression after beginning first-line therapy, KRAS status, ECOG PS, number of metastatic sites, liver only metastasis, CEA, gender, and combined prior bevacizumab use (composite subgroup). Patients from both ramucirumab and placebo arms were included in the exposure-response analysis only if they did not die or discontinue treatment prior to Day 1 Cycle 3 and had exposure data $\left(C_{\mathrm{min}, \mathrm{ss}}\right)$ available. The matching was performed separately for each of the four $\mathrm{C}_{\text {min,ss }}$ exposure quartiles (Q1-Q4) from the ramucirumab plus FOLFIRI arm (Supplemental Table 1).

To compare the two treatment arms in each of the four matched case-control groups, Kaplan-Meier curves for OS in each group are shown in Supplemental Fig. 1. Clear separation of OS curves was observed in matched Q3 and Q4, but not Q1 and Q2. As compared with matched control patients, patients in Q3 and Q4 groups showed longer survival relative to patients in $\mathrm{Q} 1$ and $\mathrm{Q} 2$ groups. This is consistent with the exposure-response association as observed earlier. In addition, the steep dose-effect relationship depicted in Supplemental Table 3 shows that the risk of death or disease 
Table 1 Exposure-response population baseline demographics and disease characteristics by ramucirumab $C_{\text {min,ss }}$ quartile

\begin{tabular}{|c|c|c|c|c|c|}
\hline & $\begin{array}{l}\text { Placebo + FOLFIRI } \\
N=481 \\
n(\%)\end{array}$ & $\begin{array}{l}\text { Ramucirumab + FOL- } \\
\text { FIRI Q1 } \\
N=106 \\
n(\%)\end{array}$ & $\begin{array}{l}\text { Ramucirumab + FOL- } \\
\text { FIRI Q2 } \\
N=106 \\
n(\%)\end{array}$ & $\begin{array}{l}\text { Ramucirumab + FOL- } \\
\text { FIRI Q3 } \\
N=106 \\
n(\%)\end{array}$ & $\begin{array}{l}\text { Ramu- } \\
\text { cirumab + FOL- } \\
\text { FIRI Q4 } \\
N=107 \\
n(\%)\end{array}$ \\
\hline \multicolumn{6}{|l|}{ Age } \\
\hline$<65$ years & $287(59.7)$ & $70(66.0)$ & $63(59.4)$ & $67(63.2)$ & $65(60.7)$ \\
\hline$\geq 65$ years & $194(40.3)$ & $36(34.0)$ & 43 (40.6) & $39(36.8)$ & $42(39.3)$ \\
\hline \multicolumn{6}{|l|}{ Gender } \\
\hline Female & $183(38.0)$ & $45(42.5)$ & $58(54.7)$ & $45(42.5)$ & $56(52.3)$ \\
\hline Male & $298(62.0)$ & $61(57.5)$ & $48(45.3)$ & $61(57.5)$ & $51(47.7)$ \\
\hline \multicolumn{6}{|l|}{ Race } \\
\hline White & $372(77.3)$ & 73 (68.9) & $70(66.0)$ & $80(75.5)$ & $87(81.3)$ \\
\hline Asian & $92(19.1)$ & $26(24.5)$ & $31(29.2)$ & $22(20.8)$ & $18(16.8)$ \\
\hline Other & $13(2.7)$ & $5(4.7)$ & $5(4.7)$ & $4(3.8)$ & $2(1.9)$ \\
\hline Missing & $4(0.8)$ & $2(1.9)$ & $0(0.0)$ & $0(0.0)$ & $0(0.0)$ \\
\hline \multicolumn{6}{|l|}{ Region } \\
\hline Europe & $216(44.9)$ & $45(42.5)$ & $47(44.3)$ & $40(37.7)$ & $52(48.6)$ \\
\hline North America & $120(24.9)$ & $24(22.6)$ & $24(22.6)$ & $33(31.1)$ & $29(27.1)$ \\
\hline Other & $145(30.1)$ & 37 (34.9) & $35(33.0)$ & $33(31.1)$ & $26(24.3)$ \\
\hline \multicolumn{6}{|l|}{ ECOG PS } \\
\hline 0 & $238(49.5)$ & $42(39.6)$ & $52(49.1)$ & $68(64.2)$ & $58(54.2)$ \\
\hline $1+$ & $243(50.5)$ & $63(59.4)$ & $54(50.9)$ & 37 (34.9) & 48 (44.9) \\
\hline Missing & $0(0.0)$ & $1(0.9)$ & $0(0.0)$ & $1(0.9)$ & $1(0.9)$ \\
\hline \multicolumn{6}{|c|}{ Time to disease progression after first-line therapy } \\
\hline$<6$ months & $111(23.1)$ & $31(29.2)$ & $22(20.8)$ & $21(19.8)$ & $21(19.6)$ \\
\hline$\geq 6$ months & $370(76.9)$ & $75(70.8)$ & $84(79.2)$ & $85(80.2)$ & $86(80.4)$ \\
\hline \multicolumn{6}{|l|}{ KRAS Status } \\
\hline Mutant & $234(48.6)$ & $52(49.1)$ & $48(45.3)$ & $59(55.7)$ & $57(53.3)$ \\
\hline Wild-type & $247(51.4)$ & $54(50.9)$ & $58(54.7)$ & $47(44.3)$ & $50(46.7)$ \\
\hline \multicolumn{6}{|c|}{ Number of metastatic sites } \\
\hline 1 & $146(30.4)$ & $35(33.0)$ & $41(38.7)$ & $29(27.4)$ & $28(26.2)$ \\
\hline 2 & $177(36.8)$ & $40(37.7)$ & $40(37.7)$ & $42(39.6)$ & $45(42.1)$ \\
\hline$\geq 3$ & $158(32.8)$ & $31(29.2)$ & $25(23.6)$ & $35(33.0)$ & $34(31.8)$ \\
\hline \multicolumn{6}{|c|}{ Liver only metastasis } \\
\hline No & 394 (81.9) & $86(81.1)$ & $82(77.4)$ & $92(86.8)$ & $91(85.0)$ \\
\hline Yes & $87(18.1)$ & $20(18.9)$ & $24(22.6)$ & $14(13.2)$ & $16(15.0)$ \\
\hline \multicolumn{6}{|c|}{ Site of primary tumor } \\
\hline Colon & $318(66.1)$ & $58(54.7)$ & $63(59.4)$ & $82(77.4)$ & $71(66.4)$ \\
\hline Rectal & $158(32.8)$ & $48(45.3)$ & $43(40.6)$ & $22(20.8)$ & $35(32.7)$ \\
\hline Colorectal & $5(1.0)$ & $0(0.0)$ & $0(0.0)$ & $2(1.9)$ & $1(0.9)$ \\
\hline \multicolumn{6}{|c|}{ Carcinoembryonic antigen } \\
\hline$<200 \mu \mathrm{g} / \mathrm{L}$ & $359(74.6)$ & $77(72.6)$ & $77(72.6)$ & $84(79.2)$ & $79(73.8)$ \\
\hline$\geq 200 \mu \mathrm{g} / \mathrm{L}$ & $91(18.9)$ & $23(21.7)$ & $23(21.7)$ & $16(15.1)$ & $21(19.6)$ \\
\hline Missing & $31(6.4)$ & $6(5.7)$ & $6(5.7)$ & $6(5.7)$ & $7(6.5)$ \\
\hline \multicolumn{6}{|c|}{ Time from first bevacizumab dose to last bevacizumab dose } \\
\hline$<3$ months & $88(18.3)$ & $13(12.3)$ & 15 (14.2) & $14(13.2)$ & $13(12.1)$ \\
\hline
\end{tabular}


Table 1 (continued)

\begin{tabular}{|c|c|c|c|c|c|}
\hline & $\begin{array}{l}\text { Placebo + FOLFIRI } \\
N=481 \\
n(\%)\end{array}$ & $\begin{array}{l}\text { Ramucirumab + FOL- } \\
\text { FIRI Q1 } \\
N=106 \\
n(\%)\end{array}$ & $\begin{array}{l}\text { Ramucirumab + FOL- } \\
\text { FIRI Q2 } \\
N=106 \\
n(\%)\end{array}$ & $\begin{array}{l}\text { Ramucirumab + FOL- } \\
\text { FIRI Q3 } \\
N=106 \\
n(\%)\end{array}$ & $\begin{array}{l}\text { Ramu- } \\
\text { cirumab + FOL- } \\
\text { FIRI Q4 } \\
N=107 \\
n(\%)\end{array}$ \\
\hline$\geq 3$ months & $391(81.3)$ & 93 (87.7) & $90(84.9)$ & $91(85.8)$ & $94(87.9)$ \\
\hline Missing & $2(0.4)^{\mathrm{a}}$ & $0(0.0)$ & $1(0.9)$ & $1(0.9)$ & $0(0.0)$ \\
\hline
\end{tabular}

Note Patients in each exposure quartile group were a non-randomized subset of the ITT population and potential imbalances in prognostic factors between the placebo arm and the quartile groups may be generated due to the loss of randomization. However, the multivariate Cox regression analysis was adjusted for all prognostic factors significantly associated with OS or PFS

$C_{\text {min,ss }}$ minimum concentration at steady state, ECOG PS Eastern Oncology Cooperative Group performance status, FOLFIRI folinic acid, 5-fluorouracil, and irinotecan; ITT intent-to-treat, $O S$ overall survival, $P F S$ progression-free survival, $Q$ quartile

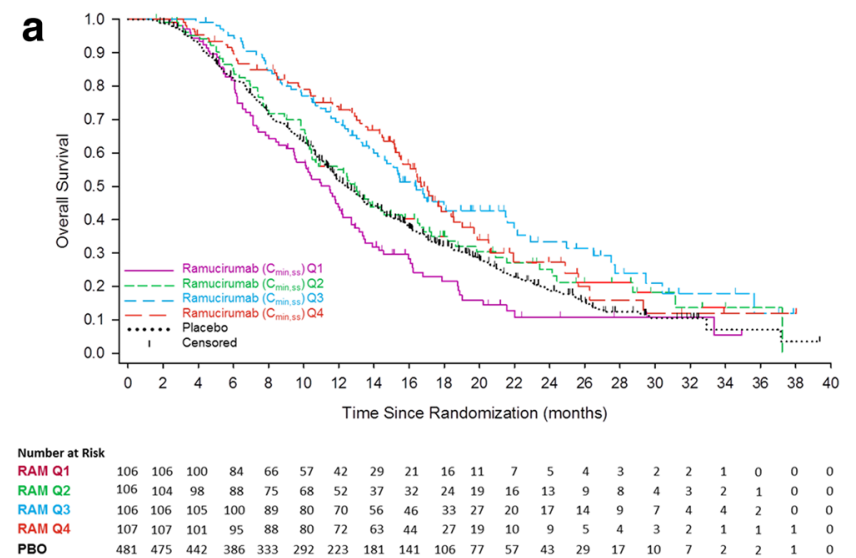

Fig. 2 a RAISE overall survival by ramucirumab $\mathrm{C}_{\min , \mathrm{ss}}$ exposure quartile. Ramucirumab $\mathrm{C}_{\text {min,ss }}$ quartile concentrations: Q1 $(<25 \%)$ $<49.7 \mu \mathrm{g} / \mathrm{mL}, \mathrm{Q} 2(25 \%$ to $<50 \%) 49.7$ to $<62.6 \mu \mathrm{g} / \mathrm{mL}, \mathrm{Q} 3(50 \%$ to $<75 \%) 62.6$ to $<81.1 \mu \mathrm{g} / \mathrm{mL}$, Q4 $(\geq 75 \%) \geq 81.1 \mu \mathrm{g} / \mathrm{mL}$. b RAISE progression-free survival by ramucirumab $\mathrm{C}_{\mathrm{min}, \mathrm{ss}}$ exposure quartile.

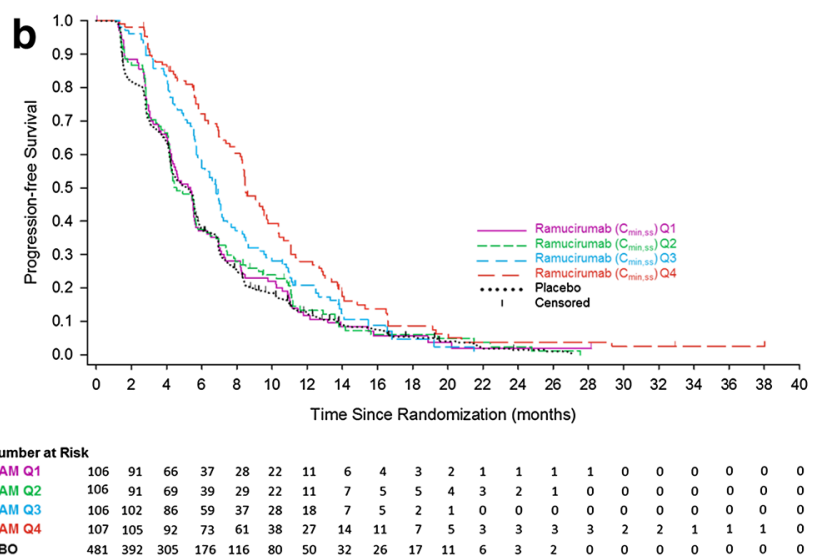

Ramucirumab $\mathrm{C}_{\min , \mathrm{ss}}$ quartile sconcentrations: Q1 $(<25 \%)<49.7 \mu \mathrm{g} /$ $\mathrm{mL}, \mathrm{Q} 2(25 \%$ to $<50 \%) 49.7$ to $<62.6 \mu \mathrm{g} / \mathrm{mL}$, Q3 $(50 \%$ to $<75 \%)$ 62.6 to $<81.1 \mu \mathrm{g} / \mathrm{mL}, \mathrm{Q} 4(\geq 75 \%) \geq 81.1 \mu \mathrm{g} / \mathrm{mL} \mathrm{C}_{\text {min,ss }}$, minimum concentration at steady state; $P B O$ placebo; $Q$ quartile; $R A M$ ramucirumab

Table 2 RAISE overall survival and progression-free survival by ramucirumab exposure quartile

\begin{tabular}{|c|c|c|c|c|c|c|}
\hline \multirow[t]{2}{*}{ Quartile, $C_{\min , \mathrm{ss}}$} & \multicolumn{3}{|c|}{ Overall survival $^{\mathrm{a}}$} & \multicolumn{3}{|c|}{ Progression-free survival $^{\mathrm{b}}$} \\
\hline & Median (mos) & Hazard ratio $(95 \% \mathrm{CI})^{\mathrm{c}}$ & $p$ value $^{\mathrm{d}}$ & Median (mos) & Hazard ratio $(95 \% \mathrm{CI})^{\mathrm{c}}$ & $p$ value $^{\mathrm{d}}$ \\
\hline PBO + FOLFIRI & $12.4^{\mathrm{e}}$ & & & $5.2^{\mathrm{e}}$ & & \\
\hline RAM + FOLFIRI Q1 & 11.5 & $1.311(1.024,1.678)$ & 0.0314 & 5.4 & $0.932(0.739,1.175)$ & 0.5516 \\
\hline RAM + FOLFIRI Q2 & 12.9 & $0.954(0.736,1.238)$ & 0.7249 & 4.6 & $0.957(0.763,1.200)$ & 0.7013 \\
\hline RAM + FOLFIRI Q3 & 16.4 & $0.604(0.459,0.795)$ & 0.0003 & 6.8 & $0.684(0.542,0.864)$ & 0.0014 \\
\hline RAM + FOLFIRI Q4 & 16.7 & $0.657(0.500,0.865)$ & 0.0027 & 8.5 & $0.546(0.434,0.688)$ & $<0.0001$ \\
\hline
\end{tabular}

$C_{\text {min,ss }}$ minimum concentration at steady state, ECOG PS Eastern Oncology Cooperative Group performance status, FOLFIRI folinic acid, 5-fluorouracil, and irinotecan, $O S$ overall survival, $P B O$ placebo, $P F S$ progression-free survival, $Q$ quartile, $R A M$ ramucirumab

${ }^{a}$ Adjusted for time to progression after beginning first-line therapy, KRAS status, ECOG PS, number of metastatic sites, liver only metastasis, and carcinoembryonic antigen

${ }^{\mathrm{b}}$ Adjusted for ECOG PS, number of metastatic sites, liver only metastasis, carcinoembryonic antigen, and prior bevacizumab use

${ }^{c}$ Adjusted for significant prognostic factors relative to PBO + FOLFIRI in RAISE

${ }^{\mathrm{d}}$ Wald's test of RAM quartile versus PBO + FOLFIRI

${ }^{\mathrm{e}}$ Median OS and PFS for PBO + FOLFIRI differ from those reported in Tabernero et al. [9], because patients in the PBO arm who dropped out prior to the third dose were excluded from the exposure-efficacy analyses 
progression was reduced by approximately $40 \%$ or $30 \%$, respectively, when $C_{\mathrm{min}, \mathrm{ss}}$ was doubled.

The percentage of patients with dose modifications (dose delay, dose reduction, and dose omission) or dose discontinuation of ramucirumab in all four ramucirumab plus FOLFIRI quartiles was generally higher as compared to the placebo plus FOLFIRI arm (Fig. 3). Patients with higher ramucirumab exposure appeared to have higher incidence of dose delay as compared to patients with lower exposure (Fig. 3). The percentage of patients with dose modifications (dose delay, dose reduction, and dose omission) or dose discontinuation of FOLFIRI in all four ramucirumab plus FOLFIRI quartiles was generally higher as compared to the placebo plus FOLFIRI arm. In addition, patients with higher ramucirumab exposure also had higher incidence of dose modifications of FOLFIRI as compared to patients with lower exposure (Fig. 3). Higher incidence of 5-fluorouracil dose discontinuation was observed in patients with higher ramucirumab exposure. Thus, although Q4 patients had more dose reduction and delay, they still had superior PFS and OS to lower exposure quartiles.

Observed TEAE incidences ( $\geq$ Grade 3 ) (selected safety endpoints for exposure-safety analysis) for both ramucirumab and placebo arms were similar between the overall study population and the exposure-response analysis population (Supplemental Table 2). Incidence differences between ramucirumab and placebo arms were also similar in these two populations. Therefore, the safety data used in the exposure-safety analysis were judged representative of data obtained from the overall study population. The observed incidences of Grade $\geq 3$ neutropenia, hypertension, fatigue, and diarrhea by concentration quartile are shown in Fig. 4. There were no Grade 5 TEAEs reported. For patients receiving ramucirumab plus FOLFIRI, neutropenia was the Grade $\geq 3$ TEAE with the highest incidence in all quartiles, and was most frequently reported in $\mathrm{Q} 4$, patients with the highest concentration of ramucirumab. A statistically significant $(p<0.001)$ relationship was only identified between $C_{\text {min,ss }}$ and incidence of neutropenia (Grade $\geq 3$ ). Severity of neutropenia (Grade 3 vs Grade 4) was independent of exposure. Additional covariate analyses found age at study entry, sex, and Asian race to be significant predictors for risk of neutropenia in CRC patients regardless of treatment arm. It is of interest to note that for neutropenia, hypertension, fatigue, and diarrhea, most of the increase in toxicity occurred between the control and Q1 and very little increase in toxicity occurred between Q1 and Q4.

\section{Discussion}

In the RAISE trial, median OS and PFS were significantly greater for patients receiving ramucirumab plus FOLFIRI when compared to placebo plus FOLFIRI treatment [9]. Exploratory exposure-response analyses presented here demonstrate that longer median OS and PFS may be associated with increasing ramucirumab exposure as seen in patients in higher exposure quartiles (Q3 and Q4). This relationship was demonstrated with both an unadjusted analysis and a matched pair analysis. These analyses suggest that the
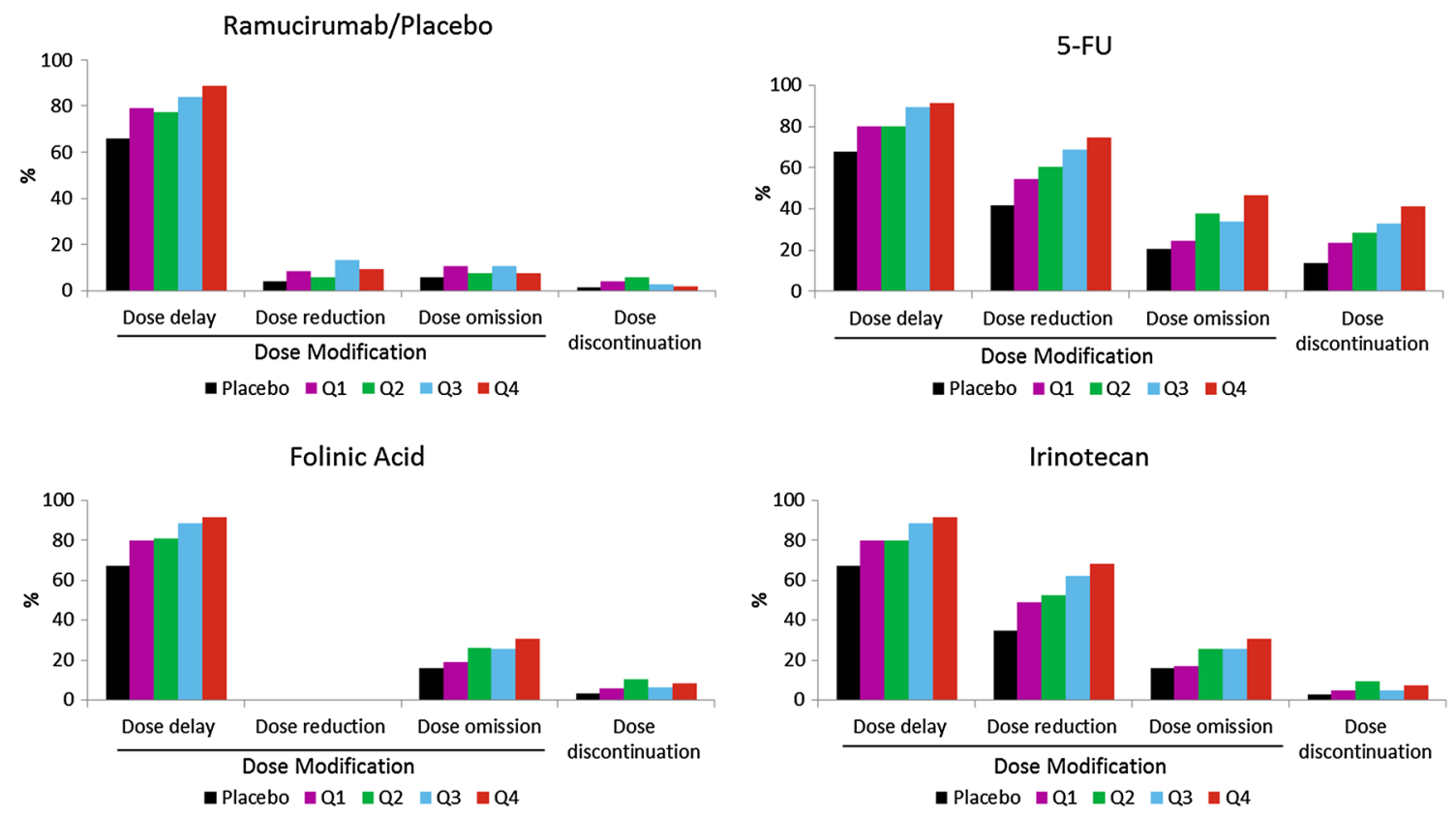

Fig. 3 Dose omission/dose modification by exposure-response quartile 5-FU, 5-fluorouracil, $Q$ quartile 


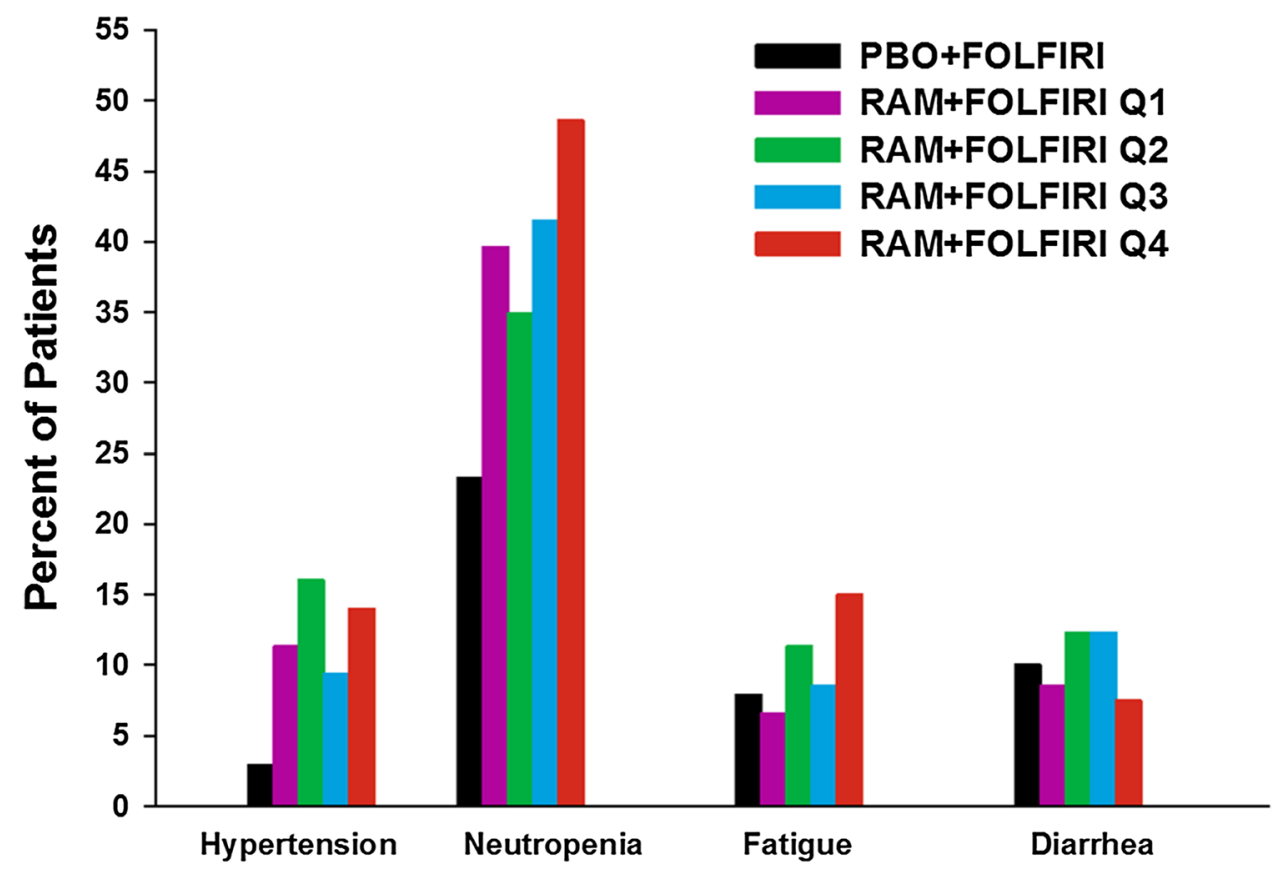

Fig. 4 Incidence of Grade $\geq 3$ treatment-emergent adverse events by ramucirumab $\mathrm{C}_{\text {min,ss }}$ exposure quartile. There was only one reported Grade 4 hypertension event, nine reported Grade 4 diarrhea events, and no Grade 4 fatigue events. A total of $9.4 \%$ patients reported Grade 4 neutropenia. There were no Grade 5 events for all four safety endpoints. Treatment-emergent adverse events were graded by NCICTCAE v4.0. Neutropenia and fatigue are consolidated terms, mean-

observed exposure-efficacy relationship is independent of a patient's baseline characteristics. Although incidence of $\geq$ Grade 3 neutropenia was found to be significantly correlated with predicted ramucirumab concentration, severity of neutropenia (Grade 3 vs Grade 4) was independent of exposure. Grade 3 or greater febrile neutropenia in the ITT population was low (ramucirumab: 18 patients, $3 \%$; placebo: 13 patients, 2\%) [9] and could not be evaluated by quartile due to the low incidence. There was no statistically significant relationship identified between ramucirumab exposure and hypertension, fatigue, or diarrhea. Thus, the analysis suggests that some of the toxicities seem to reach maximal intensity in the Q1 population and do not get worse with increasing exposure.

Of note, for both PFS and OS in multivariate Cox regression and the matched case-control analysis, patients in the two lowest exposure quartiles had no apparent benefit from adding ramucirumab to FOLFIRI; the benefit was seen in patients with higher exposure (Q3 and Q4). This steep dose-effect relationship shows that the risk of death or disease progression was reduced when $C_{\text {min,ss }}$ was doubled.

Although patients in the higher exposure quartiles appeared to have greater incidences of ramucirumab dose delay, there was no apparent relationship between ramucirumab exposure and dose reduction, dose omission, or ing they are a composite term consisting of multiple related preferred terms based on Standardized Medical Dictionary for Regulatory Activities (MedDRA) Queries and medical review. $\mathrm{C}_{\text {min,ss }}$, minimum concentration at steady state; FOLFIRI, folinic acid, 5-fluorouracil, and irinotecan; NCI-CTCAE National Cancer Institute-Common Terminology Criteria for Adverse Events; $P B O$ placebo; $Q$ quartile; $R A M$ ramucirumab

dose discontinuation of ramucirumab. Ramucirumab dose intensity was consistent across all four quartiles, demonstrating that a ramucirumab $8 \mathrm{mg} / \mathrm{kg}$ every-2-week dosing regimen is safe. Higher incidences of dose delay, dose reduction, dose omission, or dose discontinuation of all components of FOLFIRI were observed for higher exposure quartiles. However, despite these dose reductions and delays in the Q4 group, they have superior PFS and OS compared to lower quartiles.

The development and introduction of monoclonal antibodies targeting EGFR and angiogenic pathways have expanded treatment options for cancer patients [20-30]. In most cases, the dosing strategy is based on the titration of the drug to a tolerable dose. However, clinical pharmacokinetics have been used to confirm the rationale for the recommended dose of antiangiogenic agents [31-33]. The current results with ramucirumab are also consistent with exposure-response (efficacy and safety) relationships observed for agents targeting tyrosine kinase inhibitors (TKIs). Exposure-response analyses of sunitinib, an oral multi-targeted receptor TKI, demonstrated that increased exposure is associated with longer OS, greater antitumor response, longer time to tumor progression, and some increased risk of adverse effects in patients with advanced tumors [34]. Similarly, greater $C_{\min }$ of nilotinib, a selective 
break point cluster-Abelson (BCR-ABL) TKI, was associated with shorter time to complete cytogenetic response, shorter time to major molecular response, longer time to progression, and a trend toward higher response rates in patients with chronic myeloid leukemia [35]. These results are also consistent with exposure-response relationships observed for other agents (ipilimumab, ado-trastuzumab, and rituximab) targeting pathways in cancer. A higher $C_{\min }$ of ipilimumab, a fully human IgG1 monoclonal antibody that blocks cytotoxic T-lymphocyte antigen-4, was associated with increased tumor responses and longer survival in patients with advanced melanoma [10]. An association between $C_{\min }$ levels and OS, PFS, and objective response rate was observed for ado-trastuzumab, a human epidermal growth factor 2 (HER2)-directed antibody-drug conjugate, in HER-2 positive metastatic breast cancer [11]. In addition, increased clearance of bevacizumab, a humanized monoclonal IgG1 antibody that targets VEGF-A, was also associated with poorer prognosis for gastric cancer patients [36].

Previous analyses have demonstrated that higher predicted ramucirumab exposure was associated with longer OS and PFS, smaller hazard ratios, and increased but manageable toxicity in patients with previously-treated advanced gastric or gastroesophageal cancer as well as in patients with metastatic non-small cell lung cancer [13, 14]. The ramucirumab dose of $8 \mathrm{mg} / \mathrm{kg}$ every 2 weeks regimen is a clinically effective and safe dose in the CRC indication and offers a favorable benefit-risk profile in patients with CRC. The present exposure-response analysis shows a positive relationship between efficacy and ramucirumab exposure, seen particularly in the upper two quartiles. This opens the question of whether an increased dose of ramucirumab could achieve higher exposure in more patients, thus increasing the efficacy of ramucirumab treatment while maintaining a tolerable safety profile. Such clinical trials are ongoing in gastric and gastroesophageal junction cancers: with monotherapy (NCT02443883) and in the combination therapy with paclitaxel setting (NCT02514551).

Acknowledgements The study was funded by Eli Lilly. We thank the patients, investigators, and institutions involved in this study and the members of the data monitoring committee. In addition, we thank Michelle Mynderse, $\mathrm{PhD}$, for medical writing support and Casie Polanco for editorial support, both inVentiv Health Clinical and contracted by Eli Lilly and Company.

\section{Compliance with ethical standards}

Conflict of interest ALC has served in a speaker's bureau for Merrimack, and consulted for BMS and Genentech; TY has received research funding from GlaxoSmithKline K.K. and Boehringer Ingelheim $\mathrm{GmbH}$; VH received honoraria from Merck, Amgen, Roche, Sanofi, and SIRTEX, consulted or served on an advisory board for Merck, Amgen, Roche, Sanofi, and SIRTEX, received research funding from Merck, Amgen, Roche, and Sanofi, and travel accommodation expenses from Merck and Roche; RO consulted or advised Amgen, Roche and Bayer, served on a speaker's bureau from Amgen, Roche, and Eli Lilly, and received research funding from Merck; DP consulted for Eli Lilly; EVC consulted and received research funding from Eli Lilly; KY received honoraria Lilly, Chugai Pharma, Daiichi Sankyo, Yakult Honsha, Takeda, Bayer, Merck Serono, Bristol-Myers Squibb Japan, and Taiho Pharmaceutical; SL served as a consultant or advised Bayer and Amgen, and served on a speaker's bureau for Roche and Eli Lilly; JT has served on advisory boards for Amgen, Bayer, Boehringer Ingelheim, Celgene, Chugai, Eli Lilly, MSD, Merck Serono, Novartis, Pfizer, Roche, Sanofi, Symphogen, Tahio, and Takeda. LO, LG, DF, and FN are employees of Eli Lilly and Company. LMO, LG, and DF are stockholders of Eli Lilly and Company. The remaining authors had no conflicts to disclose.

Ethical approval All procedures performed in studies involving human participants were in accordance with the ethical standards of the institutional and/or national research committee and with the 1964 Helsinki declaration and its later amendments or comparable ethical standards.

Funding This study was funded by Eli Lilly and Company.

Open Access This article is distributed under the terms of the Creative Commons Attribution 4.0 International License (http://creativecommons.org/licenses/by/4.0/), which permits unrestricted use, distribution, and reproduction in any medium, provided you give appropriate credit to the original author(s) and the source, provide a link to the Creative Commons license, and indicate if changes were made.

\section{References}

1. World Health Organization (2012) GLOBOCAN 2012: Estimated cancer incidence, mortality and prevalence Worldwide in 2012. http://globocan.iarc.fr/Pages/fact_sheets_cancer.aspx. Accessed 8 June 2014

2. World Health Organization (2015) Cancer Fact sheet No. 297, updated February 2014. http://www.who.int/mediacentre/ factsheets/fs297/en/. Accessed 8 June 2015

3. Colucci G, Gebbia V, Paoletti G et al (2005) Phase III randomized trial of FOLFIRI versus FOLFOX4 in the treatment of advanced colorectal cancer: a multicenter study of the Gruppo Oncologico Dell'Italia Meridionale. J Clin Oncol 23(22):4866-4875

4. Douillard JY, Cunningham D, Roth AD et al (2000) Irinotecan combined with fluorouracil compared with fluorouracil alone as first-line treatment for metastatic colorectal cancer: a multicentre randomised trial. Lancet 355(9209):1041-1047

5. Goldberg RM, Sargent DJ, Morton RF et al (2004) A randomized controlled trial of fluorouracil plus leucovorin, irinotecan, and oxaliplatin combinations in patients with previously untreated metastatic colorectal cancer. J Clin Oncol 22(1):23-30

6. Saltz LB, Cox JV, Blanke C et al (2000) Irinotecan plus fluorouracil and leucovorin for metastatic colorectal cancer. Irinotecan Study Group. N Engl J Med 343(13):905-914

7. Tournigand C, Andre T, Achille E et al (2004) FOLFIRI followed by FOLFOX6 or the reverse sequence in advanced colorectal cancer: a randomized GERCOR study. J Clin Oncol 22(2):229-237

8. Spratlin JL, Cohen RB, Eadens M et al (2010) Phase I pharmacologic and biologic study of ramucirumab (IMC-1121B), a fully human immunoglobulin G1 monoclonal antibody targeting the vascular endothelial growth factor receptor-2. J Clin Oncol 28(5):780-787 
9. Tabernero J, Yoshino T, Cohn AL et al (2015) Ramucirumab versus placebo in combination with second-line FOLFIRI in patients with metastatic colorectal carcinoma that progressed during or after first-line therapy with bevacizumab, oxaliplatin, and a fluoropyrimidine (RAISE): a randomised, double-blind, multicentre, phase 3 study. Lancet Oncol 16(5):499-508

10. Feng Y, Roy A, Masson E, Chen TT, Humphrey R, Weber JS (2013) Exposure-response relationships of the efficacy and safety of ipilimumab in patients with advanced melanoma. Clin Cancer Res 19(14):3977-3986

11. Wang J, Song P, Schrieber S et al (2014) Exposure-response relationship of T-DM1: insight into dose optimization for patients with HER2-positive metastatic breast cancer. Clin Pharmacol Ther 95(5):558-564

12. Zhu M, Tang R, Doshi S et al (2015) Exposure-response analysis of rilotumumab in gastric cancer: the role of tumour MET expression. Br J Cancer 112(3):429-437

13. Tabernero J, Ohtsu A, Muro K et al (2016) Exposure-response (E-R) relationship of ramucirumab (RAM) from two global, randomized, double-blind, phase 3 studies of patients (Pts) with advanced second-line gastric cancer. J Clin Oncol 33[(suppl 3):abstr 121]

14. Smit EF, Perol M, Reck M et al (2015) Exposure-response relationship for ramucirumab (RAM) from the randomized, doubleblind, phase III REVEL trial (docetaxel [DOC] vs DOC plus RAM) in second-line treatment of metastatic non-small cell lung cancer (NSCLC). J Clin Oncol 33(suppl):8053

15. O’Brien L, Westwood P, Gao L, Heathman M (2015) Population pharmacokinetic meta-analysis of Ramucirumab in cancer patients. J Pharmacokinet Pharmacodyn 42(Supplement 1):S11-S107

16. U.S. Department of Health and Human Services (2003) Guidance for industry, exposure-response relationships-study design, data analysis, and regulatory applications. https://www.fda.gov/ downloads/drugs/guidancecomplianceregulatoryinformation/ guidances/ucm072109.pdf. Accessed 8 June 2015

17. Yang J, Zhao H, Garnett $C$ et al (2013) The combination of exposure-response and case-control analyses in regulatory decision making. J Clin Pharmacol 53(2):160-166

18. Shigeta K, Hasegawa H, Okabayashi K et al (2016) Randomized phase II trial of TEGAFIRI (tegafur/uracil, oral leucovorin, irinotecan) compared with FOLFIRI (folinic acid, 5-fluorouracil, irinotecan) in patients with unresectable/recurrent colorectal cancer. Int J Cancer 139(4):946-954

19. Van Cutsem E, Tabernero J, Lakomy R et al (2012) Addition of aflibercept to fluorouracil, leucovorin, and irinotecan improves survival in a phase III randomized trial in patients with metastatic colorectal cancer previously treated with an oxaliplatin-based regimen. J Clin Oncol 30(28):3499-3506

20. Elez E, Kocakova I, Hohler T et al (2015) Abituzumab combined with cetuximab plus irinotecan versus cetuximab plus irinotecan alone for patients with KRAS wild-type metastatic colorectal cancer: the randomised phase I/II POSEIDON trial. Ann Oncol 26(1):132-140

21. Gibson TB, Ranganathan A, Grothey A (2006) Randomized phase III trial results of panitumumab, a fully human anti-epidermal growth factor receptor monoclonal antibody, in metastatic colorectal cancer. Clin Colorectal Cancer 6(1):29-31
22. Grothey A, George S, van Cutsem E, Blay JY, Sobrero A, Demetri GD (2014) Optimizing treatment outcomes with regorafenib: personalized dosing and other strategies to support patient care. Oncologist 19(6):669-680

23. Grothey A (2015) Regorafenib in metastatic colorectal cancer: optimal dosing and patient selection recommendations. Clin Adv Hematol Oncol 13(8):514-517

24. Hecht JR, Patnaik A, Berlin J et al (2007) Panitumumab monotherapy in patients with previously treated metastatic colorectal cancer. Cancer 110(5):980-988

25. Lv Y, Yang Z, Zhao L, Zhao S, Han J, Zheng L (2015) The efficacy and safety of adding bevacizumab to cetuximab- or panitumumab-based therapy in the treatment of patients with metastatic colorectal cancer (mCRC): a meta-analysis from randomized control trials. Int J Clin Exp Med 8(1):334-345

26. Saltz LB, Clarke S, Diaz-Rubio E et al (2008) Bevacizumab in combination with oxaliplatin-based chemotherapy as first-line therapy in metastatic colorectal cancer: a randomized phase III study. J Clin Oncol 26(12):2013-2019

27. Van Cutsem E, Rivera F, Berry S et al (2009) Safety and efficacy of first-line bevacizumab with FOLFOX, XELOX, FOLFIRI and fluoropyrimidines in metastatic colorectal cancer: the BEAT study. Ann Oncol 20(11):1842-1847

28. Van Cutsem E, Siena S, Humblet Y et al (2008) An open-label, single-arm study assessing safety and efficacy of panitumumab in patients with metastatic colorectal cancer refractory to standard chemotherapy. Ann Oncol 19(1):92-98

29. Van Cutsem E, Lenz HJ, Kohne CH et al (2015) Fluorouracil, leucovorin, and irinotecan plus cetuximab treatment and RAS mutations in colorectal cancer. J Clin Oncol 33(7):692-700

30. Wilhelm SM, Dumas J, Adnane L et al (2011) Regorafenib (BAY 73-4506): a new oral multikinase inhibitor of angiogenic, stromal and oncogenic receptor tyrosine kinases with potent preclinical antitumor activity. Int J Cancer 129(1):245-255

31. Thai HT, Veyrat-Follet C, Mentre F, Comets E (2013) Population pharmacokinetic analysis of free and bound aflibercept in patients with advanced solid tumors. Cancer Chemother Pharmacol 72(1):167-180

32. Tabernero J, Ciardiello F, Rivera F et al (2010) Cetuximab administered once every second week to patients with metastatic colorectal cancer: a two-part pharmacokinetic/pharmacodynamic phase I dose-escalation study. Ann Oncol 21(7):1537-1545

33. Lu JF, Bruno R, Eppler S, Novotny W, Lum B, Gaudreault J (2008) Clinical pharmacokinetics of bevacizumab in patients with solid tumors. Cancer Chemother Pharmacol 62(5):779-786

34. Houk BE, Bello CL, Poland B, Rosen LS, Demetri GD, Motzer RJ (2010) Relationship between exposure to sunitinib and efficacy and tolerability endpoints in patients with cancer: results of a pharmacokinetic/pharmacodynamic meta-analysis. Cancer Chemother Pharmacol 66(2):357-371

35. Giles FJ, Yin OQ, Sallas WM et al (2013) Nilotinib population pharmacokinetics and exposure-response analysis in patients with imatinib-resistant or -intolerant chronic myeloid leukemia. Eur J Clin Pharmacol 69(4):813-823

36. Han K, Jin J, Maia M, Lowe J, Sersch MA, Allison DE (2014) Lower exposure and faster clearance of bevacizumab in gastric cancer and the impact of patient variables: analysis of individual data from AVAGAST phase III trial. AAPS J 16(5):1056-1063 